
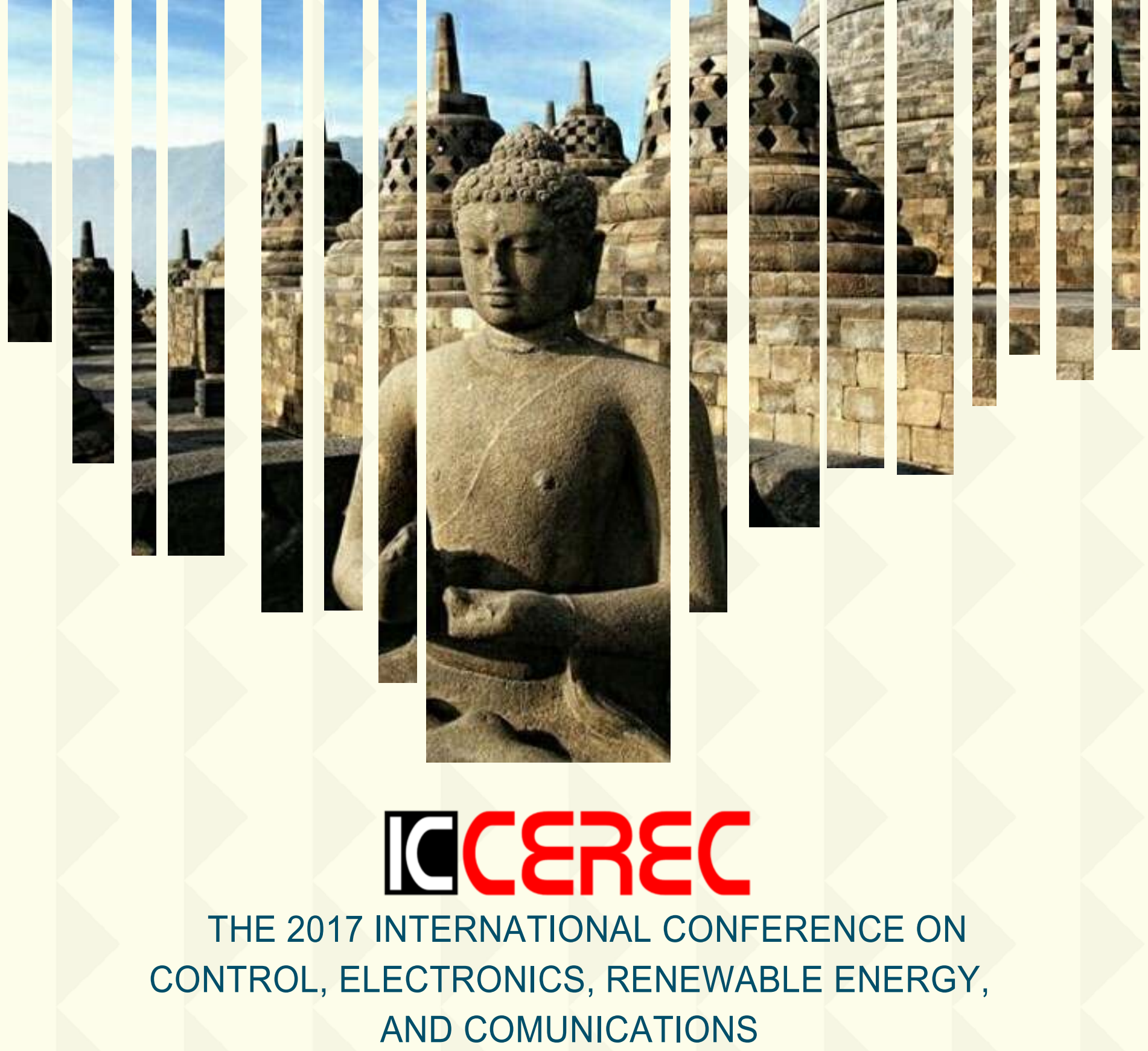

PROCEEDINGS

ISBN: $978-1-5386-1667-3$

September 26-28, 2017

Tentrem Hotel, Yogyakarta-Indonesia
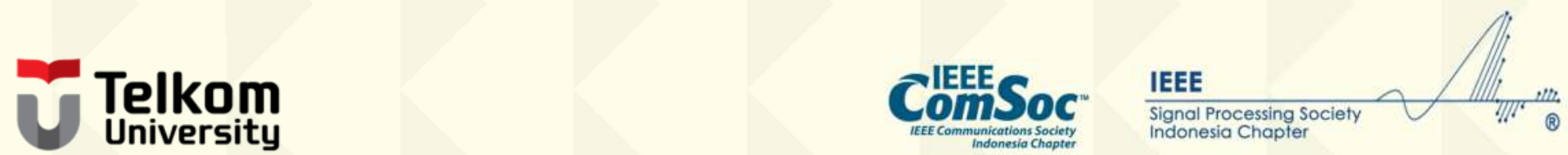

Copyright and Reprint Permission: Abstracting is permitted with credit to the source. Libraries are permitted to photocopy beyond the limit of U.S. copyright law for private use of patrons those articles in this volume that carry a code at the bottom of the first page, provided the per-copy fee indicated in the code is paid through Copyright Clearance Center, 222 Rosewood Drive, Danvers, MA 01923. For reprint or republication permission email to IEEE Copyright Manager at pubs-permissions@ieee.org All right reserved Copyright @2017 by IEEE 


\section{A B O UT \\ ICCEREC 2017}

ICCEREC 2017 is organized by the international organizing committee of ICCEREC and is technical co-sponsored by the IEEE Communications Society Indonesia Chapter and IEEE Signal Processing Society Indonesia Chapter, so that ICCEREC has a strong foundation of bringing together industry and academia.

This conference provides an international forum for researchers, academicians, professionals, and students from various engineering fields and with cross-disciplinary interests in control, electronics, renewable energy, computer engineering and communications to interact and disseminate information on the latest developments. The conference will include technical sessions, tutorials, and technology and business panels. You are invited to submit papers in all areas mentioned above. Accepted papers will be published in the ICCEREC 2017 Conference Proceedings and presented papers will be submitted to IEEE Xplore after each paper is thoroughly reviewed and (if any) satisfactorily modified according to the reviewer comments.

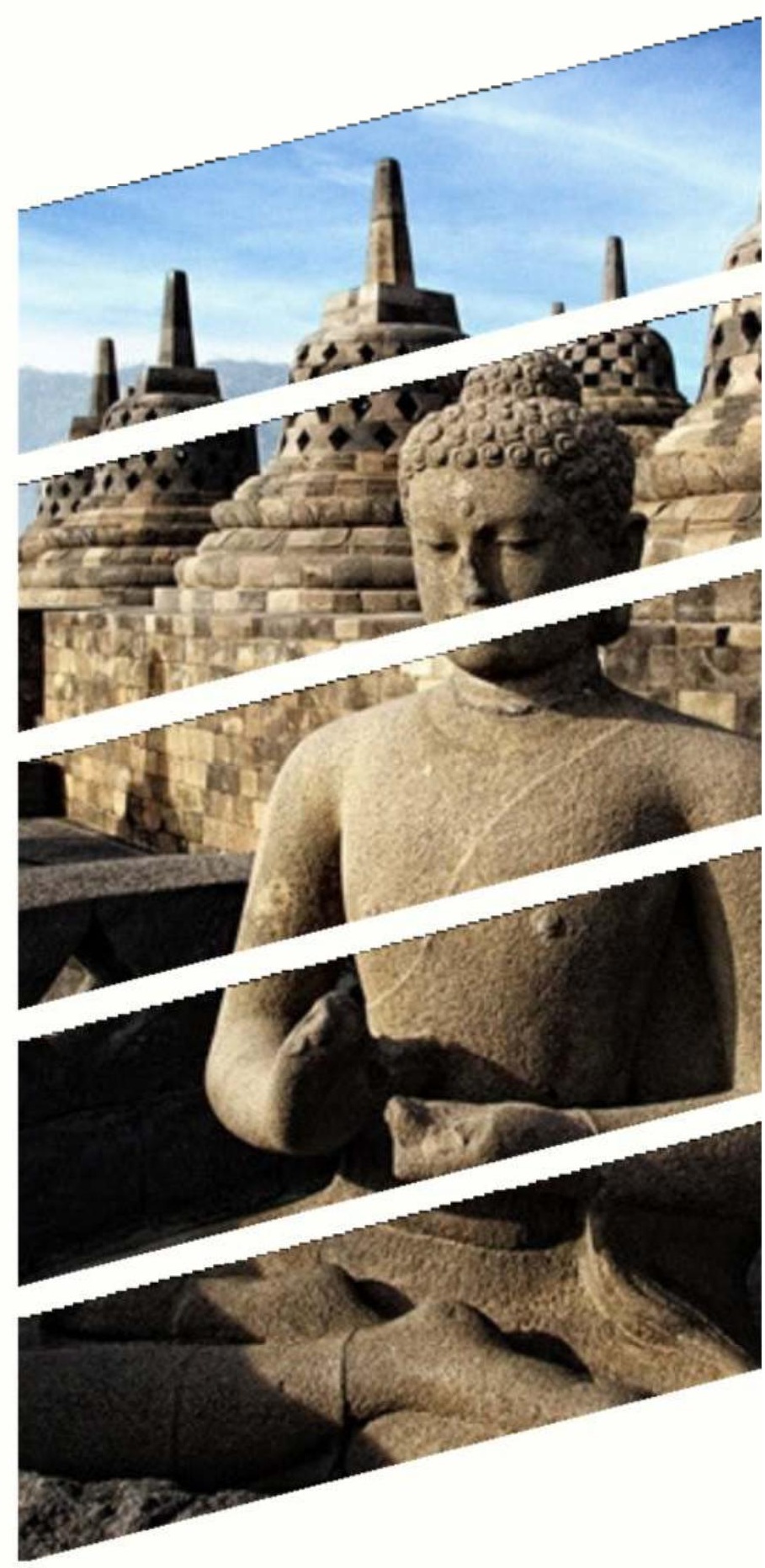




\section{WELCOMEMESSAGE}

\section{GENERAL CHAIR OF ICCEREC 2017}

\section{SIGIT YUWONO,PhD}

Welcome to ICCEREC 2017, Yogyakarta - Indonesia.

It is our great pleasure to welcome you to the International Conference on Control, Electronics, Renewable Energy, and Communications 2017 (ICCEREC 2017), which is already the 3rd running; while the 1st and the 2nd were held in Bandung in 2015 and 2016, respectively.

This conference provides an international forum for researchers, academicians, professionals, and students from various engineering fields and with cross-disciplinary interests in control, electronics, renewable energy, computer engineering and communications to interact and disseminate information on the latest developments.

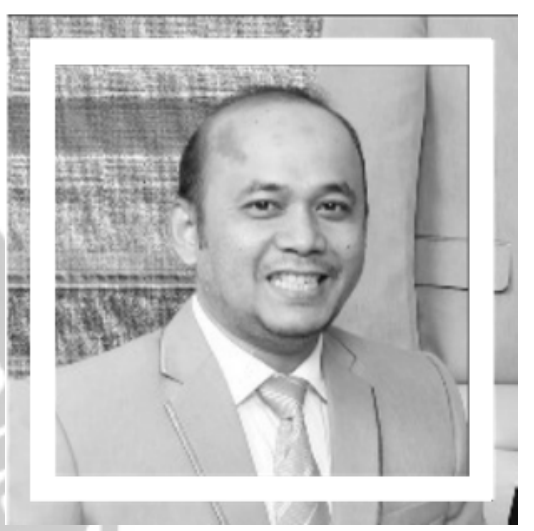

Papers submitted to ICCEREC this year came from authors in North America, Europe, Africa, and Asia countries.

ICCEREC 2017 is organized by international technical program committee, organizing committee, and international steering committee, and is technical co-sponsored by the IEEE Communications Society Indonesia Chapter and the IEEE Signal Processing Society Indonesia Chapter.

In this occasion, I would like to express my sincere appreciation to all above contributors for their great help and valuable supports to ICCEREC 2017. Many thanks to them for their efforts to bring all attendees an excellent technical program and an opportunity to spend a pleasant time at the conference.

The committee expect that the conference will bring many benefits to the scientific and technological development and to new or established international collaborations. The committee is doing its best effort for the inclusion of the conference proceedings to the IEEE Xplore Data Base. So that, the presentations of this conference will be accessible to a wider range of readers and will have continual impact to this research field.

Yogyakarta is one of the oldest cities in Indonesia, so it is very historical and is considered as the foremost cultural center of Java; therefore, tourist attractions are easily found in Yogyakarta. I hope all attendees an enjoyable and memorable stay in Yogyakarta.

Yogyakarta, 26 September 2017

Chair of ICCEREC 2017,

Sigit Yuwono, PhD.

Telkom University 


\section{TPC CHAIR OF ICCEREC 2017}

\section{Dr. RINA PUDJI ASTUTI}

Welcome to ICCEREC 2017,

It is a great honor for all of us to host of The third International Conference on Control, Electronics, Renewable Energy and Communications (ICCEREC) 2017 in Yogyakarta, Indonesia. Welcome to Yogyakarta and we hope that you enjoy the center of Javanese arts, graceful palace, the foods, and richness culture.

This conference represents a great achievement in topics of interest, which the best contributors coming from excellent laboratories and schools throughout the world, precipitate to come and contribute their finest works. Where the high qualified papers in Control, Electronics, Renewable Energy and Communicatios will be presented.

The conference received 141 papers with 350 authors from 20 countries. After carefully peer reviews by 138 reviewers, we have 68 accepted papers from 16 countries. And finally we have 50 registered papers from 10 countries. ICCEREC 2017 has maintain high quality technical program. We also would like to thank to SPS Indonesia Chapter and Telkom University as the organizer of $3^{\text {rd }}$ ICCEREC 2017, and ComSoc Indonesia Chapter that involved as Technical Co-Sponsor of the conference. We hope that fruitful discussions and exchange of ideas between researchers during conference will yield new technological innovations for contributing to a better life for humans in the coming decades.

Best Regards,

TPC Chair of ICCEREC 2017

Dr. Rina Pudji Astuti 


\section{Day One, 26 September 2017}

- $08.00-08.30$ Registration

- $08.30-09.30$ Opening Ceremony

- 09.30-10.15 Keynote Session I

- 10.15-10.30 Coffee Break

- 10.30-11.15 Keynote Session II

-11.15-12.00 Keynote Session III

- 11.00-13.00 Lunch

- 13.00-14.30 Technical Session 1 \& 2

- $14.30-14.45$ Coffee Break

- 14-45-16.30 Technical Session 3 \& 4

- 19.30-21.00 Gala Dinner

\section{Day Two, 27 September 2017}

- 08.00-09.30 Tutorial 1 \& Technical Session 5

- 09.30-09.45 Coffee Break

- 09.45-11:30 Tutorial 2 \& Technical Session 6

- 11.30-13.00 Lunch

- 13.00-14.30 Technical Session 7 \& 8

\section{Day Three, 28 September 2017}

- One Day Tour 


\section{K E Y N O T E \\ SES SIO M}

Keynote Speech 2 :

26 September 2017

$10.30-11.15$

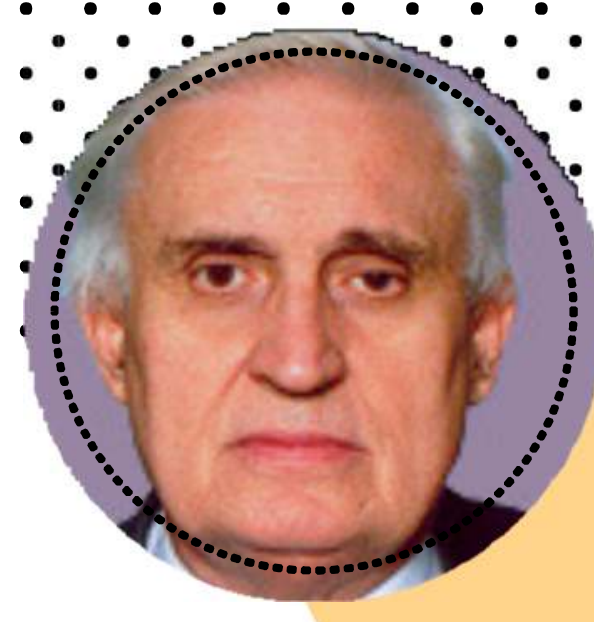

\section{Full Prof. Dr. Zoran Bojkovic}

LSM IEEE UNIVERSITY OF BELGRADE, REPUBLIC OF SERBIA

\section{"CURRENT TECHNOLOGICAL ADVANCES TO SMART GRIDS"}

Abstract:

A smart grid ( SG ) delivers electricity from suppliers to consumers using at the same time two-way digital technology that reduces cost and increases reliability and transparency. Here, communication networks play a critical role as the intelligence of this complex system is built based on information exchange across the power grid. An intelligent monitoring system that keeps track of all electricity flowing as well as the use of superconductive transmission lines for less power loss are included, too. Of course, the integration of alternative sources of electricity, such as solar and wind is welcome. These features help to promote energy independence and are a key tool in dealing with emergency resilience issues.It should be noted that the design of the communication network associated with the SG involves a detailed analysis of requirements, including choice of the most suitable technologies for each case study and the architecture for the resultant heterogeneous system. In this presentation, potential implications that current technological advances can make to SG are outlined firstly, such as big data, cloud computing and the Internet of Things ( loT ). Data analysis generated from various smart devices in the SG environment, is one of the most challenging tasks as it varies with respect to parameters such as size, volume, velocity and variety. Another big challenge in building SG often arises from the fast growing amount of data and limited communication resources. To address this issues, the concept of distributing communications architecture that implements SG communications in an efficient and cost- effective manner is provided. In that way, communication distance is shortened, so that the data will be delivered more efficiently and reliably. With the rapid development of electric vehicles ( EVs), the energy management issues in SGs integrated with Evs are attracting huge interest. This is a reason to tackle the corresponding issue. On the other hand, machine-to.machine communication is a significant part in SG networks. This improved automation results in a many heterogeneous applications. Thus, the final part is assigned to this goals. Finally,a number of open questions have been posed which will be of practical interest for further development of SGs and energy system as a whole. The end draws the conclusion of the presentation. 
Keynote Speech 3 :

26 September 2017

\section{$11.15-12.00$}

\section{Dr. Eng. Takayuki Nozaki}

Y A M A G CH I U N I E R S I T

\section{"INTRODUCTION AND RECENT RESULTS OF FOUNTAIN CODES"}

Abstract:

The reliable communication systems can be realized by error/erasure correcting codes. Fountain codes are erasure correcting codes realizing the reliable communications system for the user diagram protocol (UDP), which is used in multicasting and broadcasting.

The first topic of this talk is fundamentals of fountain code. This introduces two well-known fountain codes, namely, LT code and Raptor code, and these decoding algorithms. Moreover, we briefly introduce some applications of the fountain code.

The second topic is recent results of fountain codes. We introduce a recent code construction based on bit-level shift, that is, zigzag decodable fountain code, and its decoding algorithms.

Furthermore, we give some comparison a zigzag decodable fountain code with conventional fountain codes by theoretical analysis and computer simulations. 


\section{T U T O R I A L \\ SE S S I O N}

Tutorial 2 :

27 September 2017

$09.45-11.30$

\section{Assoc Prof. Dr. Jiwa abdullah}

FACULTY OF ELECTRICAL AND ELECTRONIC

ENGINEERING, UNIVERSITI TUN HUSSEIN ONN,

MALAYSIA

"MANET/WSN, PERSPECTIVES, ANALYSIS, EDUCATION AND RESEARCH POTENTIALS"

Abstract

The tutorial session involves the overview of the MANET and WSN which covers the various characteristics that govern the functionalities of these systems. We may cover topics such as: (1) Overview of MANET/WSN; (2) Proactive/Reactive Routing Protocol Analysis; (3) Clustering and Energy Consumption Analysis; (4) Performance analysis for 802.11/802.15.4; (5) WSN Simulation Platform based on Matlab for easy understanding to UG students. 
International Conference on Control, Electronics, Renewable Energy, and Communications 2017

\title{
PARALLEL SESSION
}

\author{
26 September 2017
}

Session $1: 13: 00-14: 30$

Tracks : COMP

Room : Bakau Room

\begin{tabular}{|c|c|c|c|}
\hline No & Time & Title & Authors \\
\hline 1 & $13.00-13.15$ & $\begin{array}{l}\text { Channel Selection for Common Spatial } \\
\text { Pattern Based on Energy Calculation of } \\
\text { Motor Imagery EEG Signal }\end{array}$ & $\begin{array}{l}\text { Hilman Fauzi, Ibrahim Shapiai, Noor } \\
\text { Akhmad Setiawan, Jafreezal Jaafar and } \\
\text { Mahfuzah Mustafa }\end{array}$ \\
\hline 2 & 13.15-13.30 & $\begin{array}{l}\text { Lie Detector with Pupil Dilation and Eye } \\
\text { Blinks Using Hough Transform and Frame } \\
\text { Difference Method with Fuzzy Logic }\end{array}$ & $\begin{array}{l}\text { Respatyadi Dwiatmojo, Muhammad } \\
\text { Nasrun and Casi Setianingsih }\end{array}$ \\
\hline 3 & $13.30-13.45$ & $\begin{array}{l}\text { Sentiment Analysis Using Multinomial } \\
\text { Logistic Regression }\end{array}$ & $\begin{array}{l}\text { Ramadhan Prakoso, Astri Novianty anc } \\
\text { Casi Setianingsih }\end{array}$ \\
\hline 4 & $13.45-14.00$ & $\begin{array}{l}\text { Indonesia Ancient Temple Classification } \\
\text { Using Convolutional Neural Network }\end{array}$ & $\begin{array}{l}\text { Kefin Danukusumo, Pranowo Pranowo } \\
\text { and Martinus Maslim }\end{array}$ \\
\hline 5 & $14.00-14.15$ & $\begin{array}{l}\text { Adaptive Multilevel Wavelet BCH Code } \\
\text { Method in the Audio Watermarking } \\
\text { System }\end{array}$ & Irma Safitri \\
\hline 6 & $14.15-14.30$ & $\begin{array}{l}\text { 3D GPU-Based SPH Simulation of Water } \\
\text { Waves Impacting on A Floating Object }\end{array}$ & Andhika Priyambada and Dede Tarwidi \\
\hline
\end{tabular}

Session $2: 13: 00-14: 30$

Tracks: COMM

Room: Bangkirai Room
No
Time
Title

\section{Authors}

$1 \quad 13.00-13.15$

$2 \quad 13.15-13.30$

$3 \quad 13.30-13.45$

$4 \quad 13.45-14.00$

$5 \quad 14.00-14.15$

$6 \quad 14.15-14.30$
Radiated Emission Test Analyzes Method to Investigate SAR

Low Cost Visible Light Communication Transceiver Prototype for Real Time Data and Images Transfer

A Smart Power Outlet for Electric Devices That Can Benefit from Real-Time Pricing Path Associativity Centralized Explicit Congestion Control (PACEC) for SDN Local Polynomial Regression Based Path Loss Estimation for Weighted Centroid Localization of Endoscopic Capsule Coupling Reduction Between Two Elements of Array Antenna Using UShaped Defected Ground Structure
Erik Madyo Putro, Budi Sulistya, Reza Septiawan, Arief Rufiyanto, Sardjono Trihatmo and Maratul Hamidah

Nenggala Yudhabrama, Inung Wijayanto and Sugondo Hadiyoso

Vikram Ramavarapu, Richard Sowers and Ramavarapu Sreenivas

Sofia Naning Hertiana, Adit Kurniawan and Hendrawan Hendrawan

Umma Hany and Lutfa Akter

Halason Nabaho, Mochamad Yunus, Edwar Edwar and Achmad Munir 
International Conference on Control, Electronics, Renewable Energy, and Communications 2017

PARALleL SESSION

\section{September 2017}

Session $3: 14: 45-16: 45$

Tracks : EL-REN

Room : Bakau Room

\begin{tabular}{|c|c|c|c|}
\hline No & Time & Title & Authors \\
\hline 1 & $14: 45-15.00$ & $\begin{array}{l}\text { Entropy Measurement as Features } \\
\text { Extraction in Automatic Lung Sound } \\
\text { Classification }\end{array}$ & $\begin{array}{l}\text { Achmad Rizal, Risanuri Hidayat and } \\
\text { Hanung Adi Nugroho }\end{array}$ \\
\hline 2 & 15.00-15.15 & $\begin{array}{l}\text { An Interfacing Digital Blood Pressure } \\
\text { Meter with Arduino-GSM Module for } \\
\text { Real-time Monitoring }\end{array}$ & $\begin{array}{l}\text { Zulfikar Ramli, Sugondo Hadiyoso and } \\
\text { Achmad Rizal }\end{array}$ \\
\hline 3 & $15.15-15.30$ & $\begin{array}{l}\text { Rehabilitation Exercise Monitoring } \\
\text { Device for Knee Osteoarthritis } \\
\text { Patients }\end{array}$ & $\begin{array}{l}\text { Mitra Mohd Addi and Nur Amirah } \\
\text { Ishak }\end{array}$ \\
\hline 4 & $15.30-15.45$ & $\begin{array}{l}\text { Feasibility Study of Ocean Wave } \\
\text { Energy for Wave Power Plant at } \\
\text { Sibolga-Tapanuli Tengah }\end{array}$ & $\begin{array}{l}\text { Riswan Dinzi, Hendrik Hutagalung and } \\
\text { Fahmi Fahmi }\end{array}$ \\
\hline 5 & $15.45-16.00$ & $\begin{array}{l}\text { Fuzzy Logic Based Active Power } \\
\text { Generation Dispatching Considering } \\
\text { Intermittent Wind Power Plants } \\
\text { Output }\end{array}$ & $\begin{array}{l}\text { Fatmawati Azis, Ardiaty Arief and } \\
\text { Muhammad Nappu }\end{array}$ \\
\hline 6 & $16.00-16.15$ & $\begin{array}{l}\text { Design of Solar Water Pumping System in } \\
\text { Urban Residential Building }\end{array}$ & $\begin{array}{l}\text { Prisma Megantoro, Danang Wijaya and } \\
\text { Eka Firmansyah }\end{array}$ \\
\hline 7 & $16.15-16.30$ & $\begin{array}{l}\text { Design of Hybrid PV-Generator-Battery } \\
\text { System for Two Kind of Loads at Aha } \\
\text { Village, Morotai Island, North Maluku }\end{array}$ & Salmon Hutapea and Agus Purwadi \\
\hline 8 & $16.30-16.45$ & $\begin{array}{l}\text { Electricity Price and Subsidy Scenario for } \\
\text { Hybrid Power Generations on Off-Grid } \\
\text { System }\end{array}$ & $\begin{array}{l}\text { Fadolly Ardin, Amien Rahardjo and } \\
\text { Chairul Hudaya }\end{array}$ \\
\hline
\end{tabular}


International Conference on Control, Electronics, Renewable Energy, and Communications 2017

\section{PARALLEL SESSION}

\section{September 2017}

Session $4: 14: 45-16: 45$

Tracks : COMM

Room : Bangkirai Room

\begin{tabular}{|c|c|c|c|}
\hline No & Time & Title & Authors \\
\hline 1 & $14.45-15.00$ & $\begin{array}{l}\text { Equivalent Circuit Analysis of Square-Loop- } \\
\text { Resonator BPF with CrossShaped I/O Coupling } \\
\text { for X-Band Frequency Application }\end{array}$ & Edwar Edwar and Achmad Munir \\
\hline 2 & $15.00-15.15$ & $\begin{array}{l}\text { Square Ring Microstrip Patch Triple Band } \\
\text { Antenna for GSM/ WLAN/ WiMAX System }\end{array}$ & $\begin{array}{l}\text { Abdulrashid Mumin, Jiwa } \\
\text { Abdullah, Rozlan Alias, Samsul } \\
\text { Haimi Dahlan and Raed } \\
\text { Abdulkareem Abdulhasan }\end{array}$ \\
\hline 3 & $15.15-15.30$ & $\begin{array}{l}\text { Dual List Interference Cancellation in Underlay } \\
\text { Cognitive Radio }\end{array}$ & $\begin{array}{l}\text { Linda Meylani, Adit Kurniawan and } \\
\text { Mohammad Sigit Arifianto }\end{array}$ \\
\hline 4 & $15.30-15.45$ & $\begin{array}{l}\text { Performance Analysis of Hybrid Optical } \\
\text { Amplifier in Long-Haul Ultra-Dense } \\
\text { Wavelength Division Multiplexing System }\end{array}$ & $\begin{array}{l}\text { Brian Pamukti Sunardi and } \\
\text { Akhmad Hambali }\end{array}$ \\
\hline 5 & $15.45-16.00$ & $\begin{array}{l}\text { Cohn Topology-based 1:8 Power Divider for S- } \\
\text { Band Array Antenna Feeding Network }\end{array}$ & $\begin{array}{l}\text { Achmad Munir, Endon Bharata } \\
\text { and Edwar Edwar }\end{array}$ \\
\hline 6 & $16.00-16.15$ & $\begin{array}{l}\text { Trilateration and Iterative Multilateration } \\
\text { Algorithm for Localization Schemes on } \\
\text { Wireless Sensor Network }\end{array}$ & $\begin{array}{l}\text { Matsna Rahman, Ratna Mayasari } \\
\text { and Ahmad Hanuranto }\end{array}$ \\
\hline
\end{tabular}

\section{September 2017}

Session 5 : 08:00-09:30

Tracks : COMP

Room : Bangkirai Room

No

Time

$1 \quad 08.00-08.15$

2

$08.15-08.30$

$3 \quad 08.30-08.45$

$4 \quad 08.45-09.00$

$5 \quad 09.00-09.15$

6

$09.15-09.30$

\section{Title}

Spatiotemporal Saliency Detection in Traffic Surveillance

Analysis of Flight Data Recorder Compression Reliability for Airplane on Demand Blackbox Data Transmission

Analysis of Cockpit Voice Recorder

Compression Reliability for Airplane on

Demand Blackbox Data Transmission Interpolating Redundant Spatial Data from

SHUMOO Boat Survey Due to the Current

Directions of Anyar River

Flood Forecasting Using Holt-Winters

Exponential Smoothing Method and Geographic Information System

Parallel Processing for Simulating Surface

Gravity Waves by Non-hydrostatic Model

Using Arakawa Grid

\section{Authors}

Wei Li, Dhoni Putra Setiawan and Hua-An Zhao

Dhipo Putra, Surya Michrandi Nasution and Fairuz Azmi

Setianto Nugroho, Surya Michrandi Nasution and Fairuz Azmi

Putu Harry Gunawan and Ketut Tomy Suhari

Mus'ab Abdurrahman, Budhi Irawan and Roswan Latuconsina

Putu Harry Gunawan and Mintho L. P. Siagian 
International Conference on Control, Electronics, Renewable Energy, and Communications 2017

\section{PARALLEL SESSION}

\section{September 2017}

Session 6 : 09:45-11:30

Tracks : COMP

Room : Bangkirai Room

\begin{tabular}{|c|c|c|c|}
\hline No & Time & Title & Authors \\
\hline 1 & 09.45-10.00 & $\begin{array}{l}\text { An Implementation of Weighted Moving } \\
\text { Average and Genetic Programming for } \\
\text { Rainfall Forecasting in Bandung Regency }\end{array}$ & $\begin{array}{l}\text { Budy Putra, Fhira Nhita, A Adiwijaya, } \\
\text { Deni Saepudin and Untari Wisesty }\end{array}$ \\
\hline 2 & $10.00-10.15$ & $\begin{array}{l}\text { Analysis Security Metric on BRO IPS } \\
\text { Based on CVSS and VEA-bility Metric }\end{array}$ & $\begin{array}{l}\text { I Made Dwi Suryadinata, Surya } \\
\text { Michrandi Nasution and Marisa } \\
\text { Paryasto }\end{array}$ \\
\hline 3 & $10.15-10.30$ & $\begin{array}{l}\text { Retinal Vessel Detection Based on Frangi } \\
\text { Filter and Morphological Reconstruction }\end{array}$ & $\begin{array}{l}\text { Hanung Adi Nugroho, Rezty Amalia } \\
\text { Aras, Tri Lestari and Igi Ardiyanto }\end{array}$ \\
\hline 4 & $10.30-10.45$ & $\begin{array}{l}\text { Computational Acceleration of Image } \\
\text { Inpainting Alternating-Direction Implicit } \\
\text { (ADI) Method Using GPU CUDA }\end{array}$ & $\begin{array}{l}\text { Mutaqin Akbar, Pranowo Pranowo and } \\
\text { Suyoto Suyoto }\end{array}$ \\
\hline 5 & $10.45-11.00$ & $\begin{array}{l}\text { Computing Two-layer SWE for Simulating } \\
\text { Submarine Avalanches on OpenMP }\end{array}$ & $\begin{array}{l}\text { Putu Harry Gunawan and Cassrio } \\
\text { Agustin Simanjuntak }\end{array}$ \\
\hline 6 & $11.00-11.15$ & $\begin{array}{l}\text { Automation System for Controlling and } \\
\text { Monitoring Ornamental Plants Using } \\
\text { Fuzzy Logic Method }\end{array}$ & $\begin{array}{l}\text { Rihla Ubudi, Budhi Irawan and Randy } \\
\text { Saputra }\end{array}$ \\
\hline 7 & $11.15-11.30$ & $\begin{array}{l}\text { Solution Path of Newton's Method for } \\
\text { Determining Epicenter Earthquake } \\
\text { Hazard in Italy } 24 \text { August } 2016\end{array}$ & $\begin{array}{l}\text { Putu Harry Gunawan and Nadzar } \\
\text { Prakoso }\end{array}$ \\
\hline
\end{tabular}


International Conference on Control, Electronics, Renewable Energy, and Communications 2017

\section{PARALLEL SESSION}

\section{September 2017}

Session $7: 13: 00-14: 30$

Tracks : COMP

Room : Bakau Room

\begin{tabular}{|c|c|c|c|}
\hline No & Time & Title & Authors \\
\hline 1 & $13.00-13.15$ & $\begin{array}{l}\text { PID Temperature Controlling of } \\
\text { Thermoelectric Based Cool Box }\end{array}$ & $\begin{array}{l}\text { Sundayani Sundayani, Dyan Sinulingga, } \\
\text { Fabiola Prasetyawati, Firmawan Palebangan, } \\
\text { Asep Suhendi, Ismudiati Puri Handayani, Tri } \\
\text { Ayodha Ajiwiguna and Indra Fathona }\end{array}$ \\
\hline 2 & 13.15-13.30 & Quadrotor Model with PD Controller & $\begin{array}{l}\text { Harits Anwar Rozi, Erwin Susanto and } \\
\text { Prasetya Dwi Wibawa }\end{array}$ \\
\hline 3 & $13.30-13.45$ & $\begin{array}{l}\text { Realization of Depth First Search } \\
\text { Algorithm on Line Maze Solver Robot }\end{array}$ & $\begin{array}{l}\text { Ahmad Syarif Hidayatullah, Agung Nugroho } \\
\text { Jati and Casi Setianingsih }\end{array}$ \\
\hline 4 & $13.45-14.00$ & $\begin{array}{l}\text { A Multi-Agent System for Solar } \\
\text { Driven DC Microgrid }\end{array}$ & $\begin{array}{l}\text { Diana Severine Rwegasira, Imed Saad Ben } \\
\text { Dhaou, Aron Kondoro, Naiman Shililiandumi, } \\
\text { Amleset Kelati, Nerey Mvungi and Hannu } \\
\text { Tenhunen }\end{array}$ \\
\hline 5 & $14.00-14.15$ & $\begin{array}{l}\text { Autonomous VTOL Design in } \\
\text { Quadcopter Using Feedback } \\
\text { Linearization and Fuzzy T-S }\end{array}$ & $\begin{array}{l}\text { Chalidia Nurin Hamdani, Mohammad Nuh } \\
\text { and Rusdhianto Efendi Abdul Kadir }\end{array}$ \\
\hline 6 & $14.15-14.30$ & $\begin{array}{l}\text { Control System Implementation and } \\
\text { Analysis for Omniwheel Vehicle }\end{array}$ & $\begin{array}{l}\text { Andra Bramanta, Agus Virgono and Randy } \\
\text { Saputra }\end{array}$ \\
\hline
\end{tabular}

Session $8: 13: 00-14: 30$

Tracks : COMM

Room : Bangkirai Room

\begin{tabular}{|c|c|c|c|}
\hline No & Time & Title & Authors \\
\hline 1 & $13.00-13.15$ & $\begin{array}{l}\text { Performance Analysis of Hybrid AF and DF } \\
\text { Protocol for Relay Networks }\end{array}$ & $\begin{array}{l}\text { Dhoni Putra Setiawan and Hua-An } \\
\text { Zhao }\end{array}$ \\
\hline 2 & $13.15-13.30$ & $\begin{array}{l}\text { Identifying } 4 \text { G Service Attributes on Customer } \\
\text { Satisfaction in Indonesia Market: Kano Model } \\
\text { Approach }\end{array}$ & $\begin{array}{l}\text { Al Bukhari Pahlevi and } \\
\text { Muhammad Suryanegara }\end{array}$ \\
\hline 3 & $13.30-13.45$ & $\begin{array}{l}\text { Performance Analysis of Message Drop Control } \\
\text { Source Relay (MDC-SR) in Maxprop DTN Routing }\end{array}$ & $\begin{array}{l}\text { Aditya Nikolas Putra, Leanna } \\
\text { Yovita and Tody Wibowo }\end{array}$ \\
\hline 4 & $13.45-14.00$ & $\begin{array}{l}\text { Antenna MIMO } 8 \times 8 \text { Array } 2 \text { Patch Rectangular } \\
\text { H-Slot for } 5 \text { G Access Radio at Frequency } 15 \mathrm{GHZ}\end{array}$ & $\begin{array}{l}\text { Adhie Surya Ruswanditya, Heroe } \\
\text { Wijanto and Yuyu Wahyu }\end{array}$ \\
\hline 5 & $14.00-14.15$ & $\begin{array}{l}\text { The Anyar River Depth Mapping from Surveying } \\
\text { Boat (SHUMOO) Using ArcGIS and Surfer }\end{array}$ & $\begin{array}{l}\text { Putu Harry Gunawan and Ketut } \\
\text { Tomy Suhari }\end{array}$ \\
\hline 6 & $14.15-14.30$ & $\begin{array}{l}\text { Leveraging Crime Reporting in Metro Manila } \\
\text { Using Unsupervised Crowd-sourced Data: A Case } \\
\text { for the iReport Framework }\end{array}$ & $\begin{array}{l}\text { Bernie S Fabito, Angelique } \\
\text { Lacasandile, Arlene Trillanes and } \\
\text { Emeliza Yabut }\end{array}$ \\
\hline
\end{tabular}




\section{PAPERS \\ INDEX}

International Conference on Control, Electronics, Renewable Energy, and Communications (ICCEREC 20 I 7 )

1 Radiated Emission Test Analyzes Method to Investigate SAR

Low Cost Visible Light Communication Transceiver Prototype for Real Time Data and Images Transfer

A Smart Power Outlet for Electric Devices That Can Benefit from Real-Time Pricing

4 Path Associativity Centralized Explicit Congestion Control (PACEC) for SDN

Local Polynomial Regression Based Path Loss Estimation for Weighted Centroid Localization of Endoscopic Capsule

Coupling Reduction Between Two Elements of Array Antenna Using U-Shaped

Channel Selection for Common Spatial Pattern Based on Energy Calculation of Motor Imagery EEG Signal

Lie Detector with Pupil Dilation and Eye Blinks Using Hough Transform and Frame Difference Method with Fuzzy Logic

9 Sentiment Analysis Using Multinomial Logistic Regression

Trilateration and Iterative Multilateration Algorithm for Localization Schemes on Wireless Sensor Network 
Entropy Measurement as Features Extraction in Automatic Lung Sound Classification

An Interfacing Digital Blood Pressure Meter with Arduino-GSM Module for Real-time Monitoring

21 Rehabilitation Exercise Monitoring Device for Knee Osteoarthritis Patients

Feasibility Study of Ocean Wave Energy for Wave Power Plant at Sibolga-

Tapanuli Tengah

Fuzzy Logic Based Active Power Generation Dispatching Considering

24 Design of Solar Water Pumping System in Urban Residential Building

Design of Hybrid PV-Generator-Battery System for Two Kind of Loads at Aha

Electricity Price and Subsidy Scenario for Hybrid Power Generations on Off-Grid System

27 Spatiotemporal Saliency Detection in Traffic Surveillance

Analysis of Cockpit Voice Recorder Compression Reliability for Airplane on Interpolating Redundant Spatial Data from SHUMOO Boat Survey Due to the Current Directions of Anyar River

Flood Forecasting Using Holt-Winters Exponential Smoothing Method and

Parallel Processing for Simulating Surface Gravity Waves by Non-hydrostatic Model Using Arakawa Grid

An Implementation of Weighted Moving Average and Genetic Programming for

Computational Acceleration of Image Inpainting Alternating-Direction Implicit (ADI) Method Using GPU CUDA

Solution Path of Newton's Method for Determining Epicenter Earthquake Hazard in Italy 24 August 2016 
Antenna MIMO 8×8 Array 2 Patch Rectangular H-Slot for 5G Access Radio at Frequency $15 \mathrm{GHZ}$

The Anyar River Depth Mapping from Surveying Boat (SHUMOO) Using ArcGIS and Surfer

46 PID Temperature Controlling of Thermoelectric Based Cool Box

47 Quadrotor Model with PD Controller

48 Realization of Depth First Search Algorithm on Line Maze Solver Robot

51 Control System Implementation and Analysis for Omniwheel Vehicle 
AUTHORS

INDEX
International Conference on Control, Electronics, Renewable Energy, and Communications (ICCEREC 20 I 7)
No

1

2

3

4

5

6

7

8

9

10
Author

A Adiwijaya

Abdulrashid Mumin

Achmad Munir

Achmad Rizal

Adhie Surya Ruswanditya

Adit Kurniawan

Aditya Nikolas Putra

Agung Nugroho Jati

Agus Purwadi

Agus Virgono

Ahmad Syarif Hidayatullah

Ahmad Hanuranto

Akhmad Hambali

Al Bukhari Pahlevi

Amien Rahardjo

Amleset Kelati

Angelique Lacasandile

Andhika Priyambada

Andra Bramanta

Ardiaty Arief

Arief Rufiyanto

Arlene Trillanes

Aron Kondoro

Asep Suhendi

Astri Novianty

Bernie S Fabito

Brian Pamukti Sunardi

Budhi Irawan

Budi Sulistya

Budy Putra

Casi Setianingsih

Cassrio Agustin Simanjuntak

Chairul Hudaya

Chalidia Nurin Hamdani

Danang Wijaya

Dede Tarwidi

Deni Saepudin

Dhipo Putra

Dhoni Putra Setiawan

Diana Severine Rwegasira

Dyan Sinulingga

Edwar Edwar

Eka Firmansyah
Page Number

169

70

$29,66,84$

93,98

221

18,75

217

247

127

265

247

88

80

212

132

252

231

60

265

116

1

231

252

236

46

231

80

159, 196

40, 46, 247

190

132

258

122

60

169

143

139, 207

252

236

$29,66,84$

122 
44 Emeliza Yabut

46 Erik Madyo Putro

48 Fabiola Prasetyawati 236

49 Fadolly Ardin 132

50 Fahmi Fahmi

51 Fairuz Azmi $\quad 143,148$

52 Fatmawati Azis 116

53 Fhira Nhita 169

54 Firmawan Palebangan 236

55 Halason Nabaho 29

56 Hannu Tenhunen 252

57 Hanung Adi Nugroho 93, 181

58 Harits Anwar Rozi 241

59 Hendrawan Hendrawan 1 (1) 18

60 Hendrik Hutagalung 111

61 Heroe Wijanto $C$

62 Hilman Fauzi 33

63 Hua-An Zhao 139,207

64 Ibrahim Shapiai 33

65 Igi Ardiyanto 181

66 I Made Dwi Suryadinata 174

67 Imed Saad Ben Dhaou 252

68 Indra Fathona 236

69 Inung Wijayanto 7

70 Irma Safitri 55

$\begin{array}{lll}71 & \text { Ismudiati Puri Handayani } & 236\end{array}$

72 Jafreezal Jaafar 33

73 Jiwa Abdullah 70

74 Kefin Danukusumo 50

75 Ketut Tomy Suhari $\quad 153,227$

76 Leanna Yovita 217

77 Linda Meylani $\quad 75$

78 Lutfa Akter 24

79 Mahfuzah Mustafa $\quad 33$

80 Maratul Hamidah 1

81 Marisa Paryasto 174

82 Martinus Maslim 50

83 Matsna Rahman 88

84 Mintho L. P. Siagian 164

85 Mitra Mohd Addi 103

86 Mochamad Yunus 29

87 Mohammad Nuh 258

88 Mohammad Sigit Arifianto 75

89 Muhammad Nappu 116

90 Muhammad Nasrun $\quad 40$

91 Muhammad Suryanegara 212

92 Mus'ab Abdurrahman 159

93 Mutaqin Akbar 185 
$95 \quad$ Naiman Shililiandumi 252

96 Nerey Mvungi

98 Noor Akhmad Setiawan

99 Nur Amirah Ishak

100 Pranowo Pranowo

50, 185

101 Prasetya Dwi Wibawa

102 Prisma Megantoro

103 Putu Harry Gunawan

$153,164,190,202,227$

104 Raed Abdulkareem Abdulhasan

Ramadhan Prakoso

106 Ramavarapu Sreenivas

107 Randy Saputra

196, 265

108 Ratna Mayasari

109 Respatyadi Dwiatmojo

40

110 Reza Septiawan

111 Rezty Amalia Aras

181

112 Richard Sowers

113 Rihla Ubudi

114 Risanuri Hidayat

115 Riswan Dinzi

116 Roswan Latuconsina

117 Rozlan Alias

118 Rusdhianto Efendi Abdul Kadir

119 Salmon Hutapea

120 Samsul Haimi Dahlan

121 Sardjono Trihatmo

122 Setianto Nugroho

123 Sofia Naning Hertiana

124 Sugondo Hadiyoso

7, 98

125 Sundayani Sundayani

236

126 Surya Michrandi Nasution

$143,148,174$

127 Suyoto Suyoto

185

128 Tody Wibowo

217

129 Tri Ayodha Ajiwiguna 236

130 Tri Lestari 181

131 Umma Hany 24

132 Untari Wisesty 169

133 Vikram Ramavarapu 11

134 Wei Li 139

135 Yuyu Wahyu $\quad 221$

136 Zulfikar Ramli 98 


\section{ORGANIZING GOMMITTEE}

\section{General Chair}

Sigit Yuwono, PhD (Telkom University, Indonesia)

\section{Co-Chair}

Muhammad Nasrun (Telkom University, Indonesia)

\section{Secretary}

Ratna Mayasari (Telkom University, Indonesia)

Casi Setianingsih (Telkom University, Indonesia)

\section{Treasurer}

Rita Magdalena (Telkom University, Indonesia)

\section{Publication}

Fairuz Azmi (Telkom University, Indonesia)

\section{TEGHICAL PROGRAM GOMMITTEE}

\section{Chairs}

Dr. Rina Pudji Astuti (IEEE ComSoc Indonesia Chapter)

Dr. Ing. Fiky Yosef Suratman (IEEE SPS Indonesia Chapter)

Dr. Muhammad Ary Murti (IEEE Indonesia Section)

\section{TPE MEMBER}

Prof. Akihiro Yamamura (Akita University, Japan) Prof. Amor Nafkha (CentraleSupelec, France) Prof. Augusto Casaca (INESC-ID, Portugal)

Prof. Bernd Wolfinger (University of Hamburg, Germany)

Prof. Bin Cao Harbin (Institute of Technology Shenzhen Graduate School, China)

Prof. Bong Jun Choi (The State University of New York (SUNY), Korea)

Prof. Calvin C K Chan (The Chinese University of Hong Kong, Hong Kong)

Prof. Carl Debono (University of Malta, Malta)

Prof. Carlos Calafate (Universidad Politécnica de Valencia, Spain)

Prof. Chuan-Ming Liu (National Taipei University of Technology, Taiwan)

Prof. Dang Hai Hoang (Ministry of Information and Communications, Vietnam)

Prof. Deepak Choudhary (LPU, India)

Prof. Eduard Babulak (Fort Hays State University, USA)

Prof. Eisuke Kudoh (Tohoku Institute of Technology, Japan)

Prof. Emilio Jiménez Macías (University of La Rioja, Spain) 
Prof. Erwin Daculan (De La Salle University - Manila, Philippines)

Prof. Farid Naït-Abdesselam (Paris Descartes University, France)

Prof. Francesco Verde (University of Napoli Federico II, Italy)

Prof. Francine Krief (University of Bordeaux, France)

Prof. Georgios Papadimitriou (Aristotle University, Greece)

Prof. Gerhard Wunder (Heisenberg Communications \& Information Theory Group, Germany)

Prof. Hakima Chaouchi (Telecom Sud Paris-Institut Mines Telecom, France)

Prof. Hans van den Berg (University of Twente, The Netherlands)

Prof. Harry Skianis (University of the Aegean, Greece)

Prof. Hiroki Tamura (University of Miyazaki, Japan)

Prof. Hsiao-Chun Wu (Louisiana State University, USA)

Prof. Hung-Yu Wei (National Taiwan University, Taiwan)

Prof. lickho Song (Korea Advanced Institute of Science and Technology, Korea)

Prof. Ikmo Park (Ajou University, Korea)

Prof. Imad Jawhar (UAE University, United Arab Emirates)

Prof. Ioannis Chatzigiannakis (Sapienza University of Rome, Italy)

Prof. Jae-Hyun Park (Chung-Ang University, Korea)

Prof. Jia Hou (Soochow University, P.R. China)

Prof. Jiahong Wang (Iwate Prefrctural University, Japan)

Prof. Jingon Joung (Chung-Ang University, Korea)

Prof. João Catalão (FEUP - Faculty of Engineering of the University of Porto, Italy)

Prof. Joel Rodrigues (National Institute of Telecommunications (Inatel), Brazil)

Prof. JongWon Kim (GIST (Gwangju Institute of Science \& Technology), Korea)

Prof. Jorge Sá Silva (University of Coimbra, Portugal)

Prof. Jungwoo Lee (Seoul National University, Korea)

Prof. Kaori Yoshida (Kyushu Institute of Technology, Japan)

Prof. Kasturi Vasudevan (Indian Institute of Technology Kanpur, India)

Prof. Klaus David (University of Kassel, Germany)

Prof. Klaus Moessner (University of Surrey, United Kingdom (Great Britain)

Prof. Koichi Asatani (Nankai University, Japan)

Prof. Ljiljana Trajković (Simon Fraser University, Canada)

Prof. Marco Listanti (University of Rome "La Sapienza", Italy)

Prof. Maurice Gagnaire (Telecom Paristech, France)

Prof. Mhamed Bakrim (University of Cadi Ayyad Marrakech, Morocco)

Prof. Mohamad Yusoff Alias (Multimedia University, Malaysia)

Prof. Muhammad Ali Imran (University of Glasgow, United Kingdom (Great Britain)

Prof. Mu-Song Chen (Electrical Engineering, Da-Yeh University, Taiwan)

Prof. Nasser-Eddine Rikli (King Saud University, Saudi Arabia)

Prof. Norman Beaulieu (Beijing University of Posts and Telecommunications BUPT, P.R. China)

Prof. Odiel Estrada Molina (University of Informatics Science, Cuba)

Prof. Osamu Ono (Meiji University, Japan)

Prof. Paul Gendron (University of Massachusetts Dartmouth, USA)

Prof. Paulo Pinto (Universidade Nova de Lisboa, Portugal)

Prof. Qing-An Zeng (North Carolina A\&T State University, USA)

Prof. Rajendra Boppana (University of Texas at San Antonio, USA)

Prof. Ray Sheriff (University of Bradford, United Kingdom (Great Britain)

Prof. Robert Morelos-Zaragoza (San Jose State University, USA)

Prof. Roberto Rojas-Cessa (New Jersey Institute of Technology, USA)

Prof. Sang-Kook Han (Yonsei University, Korea)

Prof. Satoshi Takahashi (Hiroshima City University, Japan) 
Prof. Seong-Ho Jeong Hankuk (University of Foreign Studies, Korea)

Prof. Shamik Sengupta (University of Nevada, Reno, USA)

Prof. Shingo Ata (Osaka City University, Japan)

Prof. Sokratis Katsikas (Norwegian University of Science and Technology, Norway)

Prof. Stephan Pfletschinger (Offenburg University of Applied Sciences, Germany)

Prof. Sy-Yen Kuo (National Taiwan University, Taiwan)

Prof. Terje Jensen (Telenor, Norway)

Prof. Wei-Guang Teng (National Cheng Kung University, Taiwan)

Prof. Woong Cho (Jungwon University, Korea)

Prof. Wuyi Yue (Konan University, Japan)

Prof. Xiaoqi Jia (Institute of Information Engineering, Chinese Academy of Sciences, P.R. China)

Prof. Xin-Mao Hunag (Aletheia University, Taiwan)

Prof. Xin-Wen Wu (Griffith University, Australia)

Prof. Young-Chon Kim (Chonbuk National University, Korea)

Prof. Youngnam Han (KAIST, Korea)

Prof. Yuh-Ren Tsai (National Tsing Hua University, Taiwan)

Prof. Zygmunt Haas (Cornell University, USA)

Prof. Abdel Ghani Aissaoui (University of Bechar, Algeria)

Prof. Filipe La-Gatta (Instituto Federal de Educação Ciência e Tecnologia do Sudeste de Minas

Gerais, Brazil)

Prof. Hamid Alasadi (IRAQ- BASRA, Iraq)

Prof. Koshy George (PES University, India)

Prof. Kui Xu (PLA University of Science and Technology, P.R. China)

Prof. Laizhong Cui (Shenzhen University, P.R. China)

Prof. Laurent Vermeiren (University of Valenciennes, France)

Prof. Leandro Silva (Universidade Federal de Alagoas, Brazil)

Prof. Melina Frenken (Jade University of Applied Sciences Oldenburg, Germany)

Prof. Nuno Garcia (Universidade da Beira Interior, Portugal)

Prof. Philip Moore (Lanzhou University, P.R. China)

Prof. Priya Ranjan (Amity University, India)

Prof. Valerio Scordamaglia (University of Reggio Calabria, Italy)

Dr. Hasrini Sari (Lecturer, Indonesia)

Dr. Arfianto Fahmi (Telkom University, Indonesia)

Dr. Liang-Bi Chen (Southern Taiwan University of Science and Technology, Taiwan)

Dr. Özgür Özdemir (Selcuk University, Turkey)

Dr. Abdallah Kassem (Notre Dame University, Lebanon)

Dr. Abderrahmane Lakas (UAE University, United Arab Emirates)

Dr. Agung Trisetyarso (Universitas Bina Nusantara, Indonesia)

Dr. Alban Duverdier (Centre National D'Etudes Spatiales (CNES) , France)

Dr. Alireza Ghasempour (University of Applied Science and Technology, Iran)

Dr. Amitava Mukherjee (IBM India Private Limited, India)

Dr. Andy Peng (University of Wisconsin - Stout, USA)

Dr. Angeles Vazquez-Castro (Universidad Autónoma de Barcelona, Spain)

Dr. Anna Antonyová (University of Prešov in Prešov, Slovakia)

Dr. Anthony Kearsley (National Institute of Standards and Technology, USA)

Dr. Anukram Mishra (Genus Power Infrastructures Ltd, Jaipur, India)

Dr. Arko Djajadi (Swiss German University, Indonesia)

Dr. Atta ur Rehman Khan (King Saud University, Saudi Arabia)

Dr. Charalampos Patrikakis (Piraeus University of Applied Sciences (TEI of Piraeus), Greece)

Dr. Chau Yuen (Singapore University of Technology and Design, Singapore) 
Dr. Davide Careglio (Universitat Politècnica de Catalunya, Spain)

Dr. Dhananjay Singh (Hankuk University of Foreign Studies, Korea)

Dr. Dimitrios Koukopoulos (University of Patras, Greece)

Dr. Dimitrios D. Vergados (University of Piraeus, Greece)

Dr. Dimitris Geneiatakis (DG Joint Research Centre, European Commision, Greece)

Dr. Doan Perdana (Telkom University, Indonesia)

Dr. Domenico Ciuonzo (Network Measurement and Monitoring (NM2), Naples, IT, Italy)

Dr. Dong Yang (Broadcom Corporation, USA)

Dr. El-Sayed El-Alfy (King Fahd University of Petroleum and Minerals (KFUPM), Saudi Arabia)

Dr. Emir Mauludi Husni (Institute of Technology at Bandung, Indonesia)

Dr. Eric Renault (Institut Mines-Telecom -- Telecom SudParis, France)

Dr. Felix Albu (Valahia University of Targoviste, Romania)

Dr. Fernando Boronat (Universitat Politecnica de Valencia, Spain)

Dr. Filip Idzikowski (Poznan University of Technology, Poland)

Dr. Florina Almenares (Universidad Carlos III de Madrid, Spain)

Dr. George Tambouratzis (Institute for Language \& Speech Processing, Greece)

Dr. Gianluigi Ferrari (University of Parma, Italy)

Dr. Grzegorz Debita (Wroclaw University of Technology, Poland)

Dr. Gunawan Wibisono (University of Indonesia, Indonesia)

Dr. Haikal El Abed (Technical Trainers College (TTC), Saudi Arabia)

Dr. Harco Leslie Hendric Spits Warnars (Bina Nusantara University, Indonesia)

Dr. Hemant Kumar Rath (Tata Consultancy Services, India)

Dr. Herve Rivano (Inria, France)

Dr. Hing Keung Lau (The Open University of Hong Kong, Hong Kong)

Dr. Hiromasa Habuchi (Ibaraki University, Japan)

Dr. Igor Bisio (University of Genoa, Italy)

Dr. Indra Adji Sulistijono (Politeknik Elektronika Negeri Surabaya (PENS) , Indonesia)

Dr. Iouliia Skliarova (University of Aveiro, Portugal)

Dr. Jaafar Gaber (UTBM, France)

Dr. Jingning Wang (The 54th Research Institute of China Electronics Technology Group Corporation)

Dr. John Vardakas (IQUADRAT Informatica S. L. Barcelona, Spain)

Dr. Josip Lorincz (University of Split, Croatia)

Dr. Katerina Kabassi (TEI of the Ionian Islands, Greece)

Dr. Khoirul Anwar (Telkom University, Indonesia)

Dr. Lin Cai (Illinois Institute of Technology, USA)

Dr. Lin Gao (Harbin Institute of Technology (Shenzhen), P.R. China)

Dr. Ling Tang (Aletheia University, Taiwan)

Dr. Mauro De Sanctis (University of Rome "Tor Vergata", Italy)

Dr. Md Zakirul Alam Bhuiyan (Fordham University, USA)

Dr. Michael Koch (Devolo AG, Germany)

Dr. Michael Rossberg (Technische Universität Ilmenau, Germany)

Dr. Michele Albano (CISTER/INESC-TEC, ISEP, Polytechnic Institute of Porto, Portugal)

Dr. Min Xie (Telenor Research, Norway)

Dr. Minh-Son Dao (Universiti Teknologi Brunei, Brunei Darussalam)

Dr. Mohamad Mroue (Lebanese University, Lebanon)

Dr. Mohamed El-Nemr (Tanta University, Egypt)

Dr. Mohamed Mahmoud (Tennessee Tech University, USA)

Dr. Muhammad Suryanegara (Universitas Indonesia, Indonesia)

Dr. N Nasimuddin (Institute for Infocomm Research, Singapore)

Dr. Ninoslav Marina (Princeton University, USA) 
Dr. Osmar Ogashawara (Federal University of Sao Carlos, Brazil)

Dr. Paolo Crippa (Università Politecnica delle Marche, Italy)

Dr. Paschalis Sofotasios (Khalifa University, Finland)

Dr. Pavel Loskot (Swansea University, United Kingdom (Great Britain)

Dr. Phakkharawat Sittiprapaporn (Mae Fah Luang, University Thailand)

Dr. Ping Zhou (Qualcomm, USA)

Dr. Prapto Nugroho (Universitas Gadjah Mada, Indonesia)

Dr. Qichun Wang (National University of Singapore, Singapore)

Dr. Rallis Papademetriou (University of Portsmouth, United Kingdom (Great Britain)

Dr. Ram Gopal Gupta (Ministry of Information Technology and Communications, India)

Dr. Rashid Ali (AMU Aligarh, India)

Dr. Salahuddin Zabir (National Institute of Technology, Tsuruoka College, Japan)

Dr. Sandra Sendra (Universidad de Granada, Spain)

Dr. Sanjay Singh (Manipal Institute of Technology, India)

Dr. Santiago Mazuelas (Qualcomm, USA)

Dr. Saud Althunibat (Al-Hussein Bin Talal University, Jordan)

Dr. Shyh-Lin Tsao (Cherry Tree Consulting Co. , Taiwan)

Dr. Stefan Mangold (Lovefield Wireless GmbH, Switzerland)

Dr. Takashi Kurimoto (NII, Japan)

Dr. Takuji Tachibana (University of Fukui, Japan)

Dr. Tetsuya Yokotani (Kanazawa Institute of Technology, Japan)

Dr. Tri Priyambodo (Universitas Gadjah Mada, Indonesia)

Dr. Vincenzo Eramo (University of Rome "La Sapienza", Italy)

Dr. Waail Al-waely (Griffith University / School of Engineering, Australia)

Dr. Wei Zhong (College of Communications Engineering, PLAUST, P.R. China)

Dr. Xinrong Li (University of North Texas, USA)

Dr. Yasin Kabalci (Nigde University, Turkey)

Dr. Zhenchuan Chai (SK HYNIX MEMORY SOLUTION, USA)

Dr. Zhiqiang Wu (Wright State University, USA)

Dr. Abrar Ismardi (Telkom University, Indonesia)

Dr. Afaf Merazi (Faculty of Exact Sciences. Djillali Liabes University of Sidi Bel Abbes, Algeria)

Dr. Akash Singh (IBM, USA)

Dr. Alessandro Bazzi (CNR, Italy)

Dr. Alexandre Guitton (Clermont University, France)

Dr. Ali Humos (Jackson State University, USA)

Dr. Aniello Castiglione (Università di Salerno, Italy)

Dr. Arianna D'Ulizia (CNR, Italy)

Dr. Asep Suhendi (Telkom University, Indonesia)

Dr. Biju Issac (Teesside University, Middlesbrough, United Kingdom (Great Britain)

Dr. Burhan Gulbahar (Ozyegin University, Turkey)

Dr. Charalampos Pitas (National Technical University of Athens, Greece)

Dr. Fernando Cerdan (Technical University of Cartagena, Spain)

Dr. Francisco Bellido-Outeiriño (University of Córdoba, Spain)

Dr. Galymzhan Nauryzbayev (Hamad Bin Khalifa University (HBKU), Qatar)

Dr. Gayan Amarasuriya (Southern Illinois University, USA)

Dr. Giovanni Barroso (Universidade Federal do Ceará, Brazil)

Dr. Hamed Mojallali (University of Guilan, Iran)

Dr. Heba Shaban (Arab Academy for Science, Technology \& Maritime Transport, Egypt)

Dr. Hengky Susanto (Huawei Technology, Hong Kong)

Dr. Indrabayu A (Hasanuddin University, Indonesia 
Dr. Joaquin Perez (Universitat Politecnica de Valencia, Spain

Dr. Julius Eiweck (NOKIA Austria, Austria)

Dr. Kannan Thirugnanam (Singapore University of Technology and Design, Singapore)

Dr. Kashif Saleem (King Saud University, Saudi Arabia)

Dr. Khanh Pham (Air Force Research Laboratory, USA)

Dr. Levy Nur (Telkom University, Indonesia)

Dr. M. Udin Harun Al Rasyid (Politeknik Elektronika Negeri Surabaya (PENS), Indonesia)

Dr. Manoj Daigavane (G H Raisoni Institute of Engineering \& Technology Nagpur, India)

Dr. Mansoor Khan (COMSATS Institute of Information Technology, Pakistan)

Dr. Matteo Petracca (Scuola Superiore Sant'Anna di Pisa, Italy)

Dr. Mehdi Taassori (Eastern Mediterranean University, Turkey)

Dr. Mehran Mazandarani (Ferdowsi University of Mashhad, Iran)

Dr. Mohammad Khalily Dermany (Islamic Azad University, Khomein Branch, Iran)

Dr. Mohammad Shojafar (University of Rome Tor Vergata, Italy)

Dr. Nasseer Bachache (University College of Humanity Studies UCH / Kufa-Iraq, Iraq)

Dr. Natapon Pantuwong (King Mongkut's Institute of Technology Ladkrabang, Thailand)

Dr. Natarajan Meghanathan (Jackson State University, USA)

Dr. Nikolaos Doukas (Hellenic Army Academy, Greece)

Dr. Omid Mahdi Ebadati E. (Hamdard University, India)

Dr. Radu Vasiu (Politehnica University of Timisoara, Romania)

Dr. Salah Benbrahim (Ecole Polytechnique, Canada)

Dr. Sang C. Lee (DGIST, Korea)

Dr. Sanjeev Metya (National Institute of Technology Arunachal Pradesh, India)

Dr. Sanyog Rawat (Manipal University Jaipur, India)

Dr. Satya Kumara (Udayana University, Indonesia)

Dr. Son Kuswadi (Politeknik Elektronika Negeri Surabaya, Indonesia)

Dr. Soo King Lim (Universiti Tunku Abdul Rahman, Malaysia)

Dr. Sotiris Karachontzitis (University of Patras, Greece)

Dr. Subhasis Bhattacharjee (Adobe Systems India Private Limited, India)

Dr. Sudhir Routray (CMR Institute of Technology, Bangalore, India)

Dr. Victor Hinostroza (Universidad Autónoma de Ciudad Juárez, Mexico)

Dr. Vijender Solanki (Anna University, Chennai, India)

Dr. Visvasuresh Victor Govindaswamy (Concordia University, USA)

Dr. Vithyacharan Retnasamy (University Malaysia Perlis, Malaysia)

Dr. Wei Feng (Tsinghua University, P.R. China)

Dr. Yancho Todorov (Aalto University, Finland)

Dr. Yingjie Wang (Shanghai Jiaotong University, P.R. China)

Dr. Yohanes Baptista Dafferianto Trinugroho (Add Energy, Norway)

Dr. Youssef Said (Tunisie Telecom, Tunisia)

Dr. Zaid Shamsan (Wireless Communication Center, Malaysia)

Dr. Zuhairiah Zainal Abidin (Universiti Tun Hussein Onn Malaysia, Malaysia) 


\section{Indonesia ancient temple classification using convolutional neural network}

\author{
Kefin Pudi Danukusumo \\ Atma Jaya Yogyakarta University \\ Yogyakarta, Indonesia \\ kefinpudi@gmail.com
}

\author{
Pranowo \\ Atma Jaya Yogyakarta University \\ Yogyakarta, Indonesia \\ pran@uajy.mail.ac.id \\ Martinus Maslim \\ Atma Jaya Yogyakarta University \\ Yogyakarta, Indonesia \\ martinusmaslim@mail.uajy.ac.id
}

\begin{abstract}
This paper describes the use of convolutional neural network(CNN) method to classify various image and photo of Indonesia ancient temple. The method itself implements Deep Learning technique designed for Computer Vision task. The idea behind CNN is image pre-processing through a stack of convolution layers to create many patterns that can be easily recognized. The result shows that the learning model has an accuracy of $98,99 \%$ on the training set and accuracy of $85.57 \%$ on the test set. With GPU performance, the time used to train the model is 389.14 seconds.
\end{abstract}

Keywords-Deep Learning; image classification; GPU; CNN; Indonesia ancient temple.

\section{INTRODUCTION}

Ancient temple in Indonesia is an essential part of a cultural image for the country. The temple uniqueness shows that the cultural and religious value could survive the test of time. Such majestic heritage surely cannot be ignored and forgotten. Therefore, to have a machine classify these temples is one of many methods to cultivate a culture with current technology.

Technology like Artificial Intelligence and Computer Vision could help us identify the right temple with precision. However, to reach a good precision, a machine has to go through a very complicated task. Various shape and material on the temple can be a very challenging and a hard task for a machine to classify. This problem mainly occurs because of a limited dataset to feed on the model and could result in a bad accuracy. Using Convolutional Neural Network (CNN) model to make machine learns features from the image will certainly improve how the machine could classify the temple images with a good accuracy.

$\mathrm{CNN}$ is a neural network method to make the model learn better to classify or recognize a visual pattern. This network is made with the assumption that the input used is an image. On each layer of convolution, the input image will be processed with many filters and will create a stack of activation map. These activation maps will create many patterns from some parts in the image that will be easier to classify. The classification progress includes the connection to neurons or activation units just like a simple neural network model. This technique can make the model learn more efficiently and greatly reduce the number of parameters in the network [1] [2]. However, using only CNN model might still result in overfitting the model. This problem could potentially make the model cannot predict the future data very well. Many solutions can solve the case of overfitting. Preprocessing image such as rotating or horizontal flip before inputted to the model is one of the better solutions, especially when the data in the dataset is too little. Another solution is to lower the model complexity with a lot of regularizations. In this paper, we implement the $\mathrm{CNN}$ method using image preprocessing, Dropout regularization, Softmax classifier and stochastic gradient descent.

The whole concept to use CNN method is a direct approach to implements Deep Learning principles, as the method itself uses a lot of layers. More layers that will be used in the model will make the network architecture to go deeper, thus requiring a bigger computation time. However, this is not a problem anymore because of GPU performance. The time required for training is why Deep Learning technique largely influenced by stronger computer, large datasets and algorithm to train deeper networks [3].

\section{THEORY}

\section{A. Convolutional Neural Network}

$\mathrm{CNN}$ is a neural network devoted to processing data that has a grid structure. The basic example of this model input is a twodimensional image. This neural network model is first successfully applied in 1998 by Yann LeCun, where he classifies the MNIST digit to document recognition [4]. The convolution name itself is a linear algebra operation that multiplies the matrix of the filter with the image to be processed. This process is called convolution and one of many types of layers in the model. Convolution layer is the most important layer to use in the model. Another layer is the Pooling Layer, which is the layer used to extract the maximum value or the average value of the parts of the pixels in the image. Here's an overview of the Convolution Neural Network architecture. 


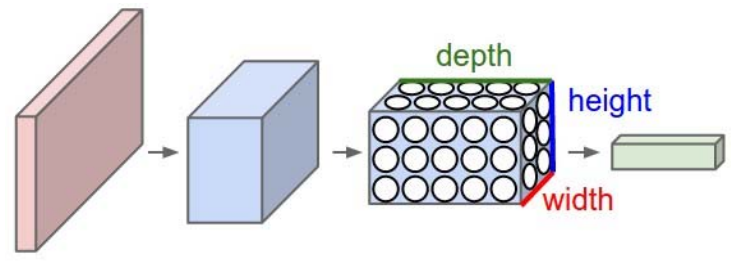

Fig.1. Convolution Neural Network Architecture Image Source: http://cs231n.github.io

From the picture above each layer has a different volume and is represented with depth, height, and width. The left red block is the input image before being processed with the filter. After the image filtered with convolution, it results in another block with reduced spatial size but deeper depth. The depth is obtained from a stack of activation map from the inputted image with different filters. The next layer shows the block with different size until the last layer where the block converted into 1dimensional and can be seen as a normal row of neurons which can be connected to the outputs. The quantity gained from each block depends on the filtration result of the previous layer and also the number of filters used. The more filters used in the model will make the depth to be deeper.

$\mathrm{CNN}$ works just like a regular neural network but with a different connection from each neuron. A regular neural network uses all connection from the previous neuron to each neuron in the next layer. CNN only uses connection to a certain local region in the next layer depending on the filters used. The local region is the activation map that comes from convolution process and creates the parameters that will determine the end result.

This neural network model has proven to be very powerful in addressing image classification problem. In 2012 Alex Krizhevsky achieved a $15.3 \%$ test error on the ImageNet dataset [1]. It shows that visual classification using CNN method is very effective.

\section{B. Convolution Operation}

Convolution operation is an operation on two functions of a real valued argument [3]. The operation is to applicate an output function as a feature map from an input image. These inputs and outputs can be seen as the two real valued arguments. Formally the operation can be written as in

$$
s(t)=(x * w)(t)
$$

The function $s(t)$ provides a single output as feature map, the first argument is the input which is $\mathrm{x}$ and the second argument $\mathrm{w}$ as the kernel or filter. If we look the input as a 2D image, then we can assume $\mathrm{t}$ as pixel and substitute it with $i$ as row and $j$ as column. Alternatively, convolution operation can be treated as a matrix multiplication between the input image and the kernel. Where the output was supposedly computed by dot product.

We can also determine the output volume from each convolution layer with hyperparameters. The hyperparameter is used in below equation to calculate how many neural activations in a single output.

$$
(W-F+2 P) / S
$$

We can compute the spatial size of the output volume as a function of the volume size $(\mathrm{W})$, the filter $(\mathrm{F})$, the stride applied $(\mathrm{S})$ and the amount of zero padding used $(\mathrm{P})$. Stride is the amount we slide the filter through the input image and zero padding is to put zeros around the image border.

\section{Pooling Layer}

A pooling layer is a function with feature map as the input and processed them with a summary statistic of the nearby outputs. Practically in a CNN model, pooling layer usually inserted regularly after a few convolution layer. Pooling layer between successive convolution layers in a CNN architecture could progressively reduce the volume size of output or feature map, thus reducing the amount of parameters and computation in the network, and to control overfitting as well. It is worth noting that choosing many types of Pooling layer can also benefit the model performance [5]. The pooling layer works on every single stack of the feature map and reduces its sizes. The most common form of pooling layer is with filters of size $2 \times 2$ applied with a stride of 2 and then operates on every slice from the input. This form will reduce the activation map to $75 \%$ from the original size.

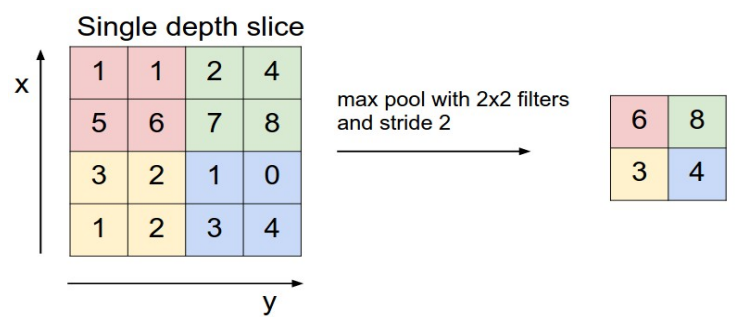

Fig.2. Max Pooling Example Image Source: http://cs231n.github.io

Pooling layer will operate on every single depth slice of the input volume. In the picture above, the pooling layer uses one of max operation which is the most common operation. Figure 2 shown the operation with a stride of 2 and pool size of $2 \times 2$. From $4 \times 4$ input size, on each 4 numbers in the input, the operation takes its maximum value and create a new slice size of $2 \times 2$.

\section{Fully-Connected Layer}

Fully-Connected layer(FC) is the layer where all the activations from the previous layer have full connections to the neuron in the next layer just like a regular neural network. Every activation from the previous layer needs to be transformed into one-dimensional data before it can be connected to all the neuron in FC layer. FC layer usually used in Multi-Layer Perceptron application and aims to process the data so it can be linearly classified.

The difference between FC and convolution layers is that the neurons in the convolution layer are connected only to a local region in the input and that many of the neurons in a convolution volume share parameters, while FC layer has the whole neuron connected. However, both layer still operate dot products, making their function not so different. 


\section{E. Dataset}

The dataset used in this paper contains the image of several ancient temples in Indonesia with the size 150 X 150 pixels. This dataset was categorized into 6 temples. The temples are Borobudur temple, Prambanan temple, Kalasan temple, Sewu temple, Mendut temple and Sari temple. This dataset has 520 training images and 225 test images collected from Flickr repository. Here is the example from each category:

\section{a. Candi Borobudur}

Borobudur temple category has 100 training images and 50 testing images.

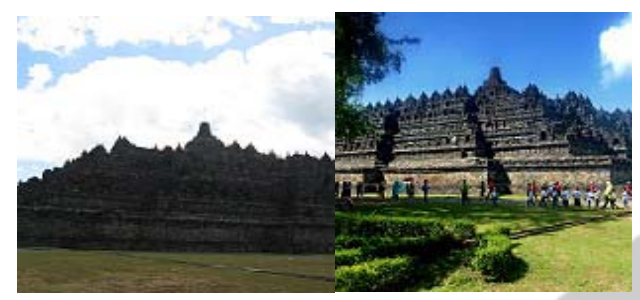

Fig.3. Borobudur Temple Image Sample Image source: http://www.flickr.com

\section{b. Candi Prambanan}

Prambanan temple category has 100 training images and 50 testing images

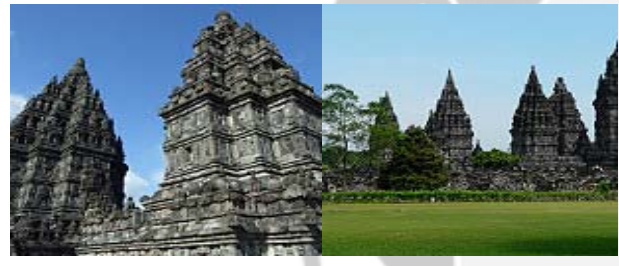

Fig.4. Prambanan Temple Image Sample Image source: http://www.flickr.com

c. Candi Kalasan

Kalasan temple category has 70 training images and 15 testing images

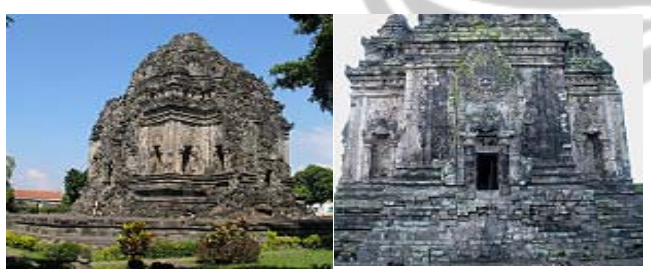

Fig.5. Kalasan Temple Image Sample Image source: http://www.flickr.com

\section{d. Candi Sewu}

Sewu temple has 100 training images and 50 testing images

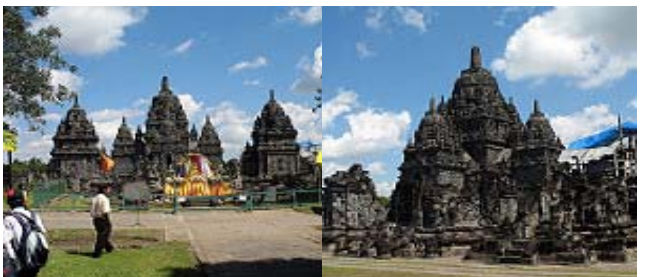

Fig.6. Sewu temple image sample Image source: http://www.flickr.com

\section{e. Candi Mendut}

Mendut temple has 100 training images and 50 testing images

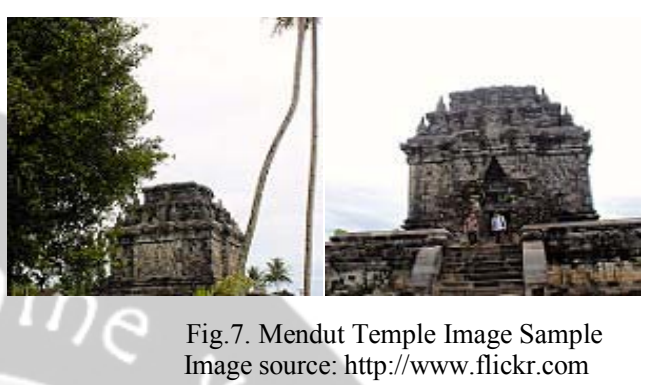

\section{f. Candi Sari}

Sari temple has 50 training images and 10 testing images

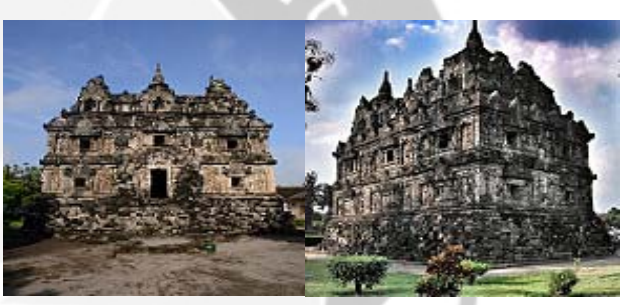

Fig.8. Sari Temple Image Sample Image source: http://www.flickr.com

\section{ALGORITHM}

In the training algorithm there are 5 main stages, namely preprocessing stage, load training data, training data, and save final weights. During pre-processing stage, image data on the dataset processed in order to increase the amount of data to be trained, for example, the image can be in rotated, horizontally and vertically reversed. This stage can potentially reduce the case of overfitting. Next, in the load training stage, the data in the dataset split into input and output. The next step is the model training stage, which is the training process by entering the pre-processed data to the pre-arranged network model. The training process will run according to how many epoch and batch size are used. After the training process is completed the final weight will be stored for testing purposes.

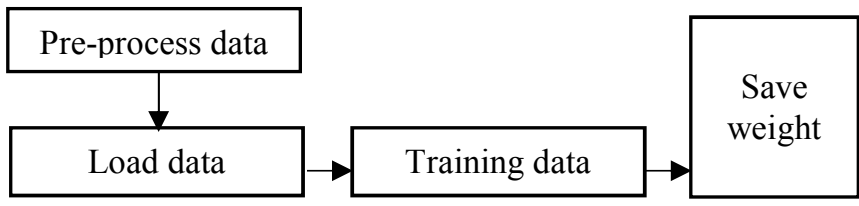

Fig.9. Training Algorithm Flow 
For the testing algorithm, there are 3 main stages, load model stage, load weight, and validation. The load stage model is the stage where CNN model is loaded as the basis for testing. Then at the load weight stage, the weights already stored in the previous training process will be used as the main parameters and will be inputted into the $\mathrm{CNN}$ model. Finally, in the validation stage, the network model with the training weights tested with the data in the test set from the dataset. The concepts used by validation basically compare the accuracy of the actual results of the test labels with the prediction given by the model, then from all the comparisons obtained, it will result in an average accuracy.

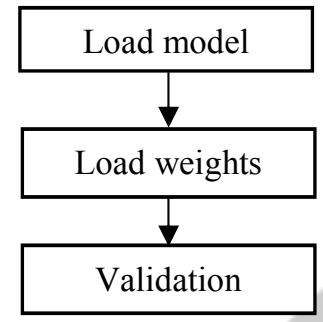

Fig.10. Testing Algorithm Flow

For the CNN model, this paper uses the following layer structure:

\section{$\mathrm{INPUT} \rightarrow \mathrm{CONV} \rightarrow \mathrm{CONV} \rightarrow \mathrm{POOL} \rightarrow \mathrm{CONV} \rightarrow \mathrm{CONV} \rightarrow$$$
\mathrm{POOL} \rightarrow \mathrm{CONV} \rightarrow \mathrm{CONV} \rightarrow \mathrm{POOL} \rightarrow \mathrm{FC}
$$

CONV is the convolution layer, POOL is the max pooling layer and FC is the Fully Connected layer. The model uses Softmax classifier to determine the class prediction. This function also trained alongside cross-entropy loss function to give the model a nonlinear variant of multivariate output. Each CONV layer also has ReLU(Rectified Linear Unit) activation function, this activation can lead to better performance improvements in deep neural networks without significantly increasing the number of parameters [6] The entire series of layers will be optimized through Stochastic Gradient Descent in order to minimize the error and effectively update the parameters in the model. Throughout the stack of layers, a dropout regularization also inserted to prevent overfitting in a powerful manner yet easy to use. [7]

\section{RESUlT}

\section{Training result}

The following analysis will illustrate how the training results from different epochs can affect the training outcomes. The training uses the training set from the dataset, the results can be compared with the following table.

\begin{tabular}{|c|c|c|}
\hline Epoch & Accuracy & Time \\
\hline 10 & $85.48 \%$ & 86.77 seconds \\
\hline 20 & $95.67 \%$ & 160.27 seconds \\
\hline 50 & $98.99 \%$ & 389.14 seconds \\
\hline
\end{tabular}

\begin{tabular}{|c|c|c|}
\hline Epoch & Accuracy & Time \\
\hline 100 & $100 \%$ & 759.27 seconds \\
\hline
\end{tabular}

Fig.11. Training Result Comparison Table

From the table above, it can be seen that the greater the epoch used for training, the training time will be longer. This obviously occurs, because with more epoch being used, there will be more training in one forward feed. One epoch means that the model trained using the entire training set in the dataset.

Judging from the accuracy side, each epoch gives a different result. There is a significant difference between epoch 10 and 20 , which is about $10 \%$ in between. But between the epochs 20 , 50 and 100 did not show a very large difference in accuracy. Only with the epoch of 20, the model can provide accuracy of $95.67 \%$. When the epoch is raised to 50 , the model gives an accuracy of $98.99 \%, 3 \%$ more than the previous one. When using the epoch of 100 , the model gives the optimal results of $100 \%$. Although accuracy increases with many epoch, from the result it is evident that the use of a very large epoch is not always optimal if we also pay attention to the time for training.

\section{Testing result}

The following analysis will describe the test results from different training weights. The training weights that used for the test is the result from different training epoch and will be tested with the test set in the dataset, the results can be compared with the following table.

\begin{tabular}{|c|c|c|}
\hline Epoch & Accuracy & Time \\
\hline 10 & $74.68 \%$ & 4.46 seconds \\
\hline 20 & $78.36 \%$ & 4.53 seconds \\
\hline 50 & $85.57 \%$ & 4.39 seconds \\
\hline 100 & $86.28 \%$ & 4.67 seconds \\
\hline
\end{tabular}

Fig.12. Testing Result Comparison Table

From the table above, it can be seen that the testing time of each epoch is not changed much. This suggests that the weight training generated from a different epoch, will not affect the time to test the model. Another reason why it does not affect time can be seen from the model used because the model used in each test is the same. All training weights have the same number of parameters as long as the same model is used, which differ only the parameter values.

Models that have been trained show better predictions than just guessing (16\%). The model with training weight of 10 epoch to 100 epoch has the accuracy exceeding $70 \%$. Obviously, with more epochs, the accuracy is also better. With every epoch that increases in number, the accuracy also increases. In tests with 10 epoch training weights, the model provides an accuracy that is not much different from that of 20 epoch, but when using 50 epoch, the accuracy is increased considerably. This suggests that large training results may not necessarily result in large predictions as well, judging by the 
case with 20 epoch where the training results reached $95.67 \%$ but with the test results only reaching $78 \%$. Whereas for 50 epoch with $98.99 \%$ training result, it can have the test result up to $85,57 \%$. In the 100 epoch test, the test results also did not show significant improvement compared with only 50 epoch, which differed only $0.69 \%$. With this result, it can be concluded that training with 50 epoch is the optimal option for training judging from the test accuracy and training time.

With this result, it can be concluded that image classification of Indonesia ancient temple using CNN model is very effective. As it can reach about $85 \%$ accuracy in clasify the test set. If we compare the test result using CIFAR-10 dataset with the same architecture model and the same pre-processing method, there are no much difference with the accuracy. The result with CIFAR-10 test set also shows about $85 \%$ accuracy.

\section{GPU vs CPU}

The model also trained using CPU and the result is the big difference in time compared with the GPU. The accuracy obtained more or less the same when using GPU. The time used to train data with CPU is 10,573 seconds using 10 epoch only, while using GPU only need 86.77 seconds. Training model using GPU almost 120 times faster than CPU. This result shows that training CNN model with GPU significantly increases performance to reduce the time.

\section{ACKNOWLEDGMENT}

The under graduate research project was supported by Data Structure Laboratory of Atma Jaya Yogyakarta University.

\section{REFERENCES}

[1] A. Krizhevsky, I. Sutskever and G. E. Hinton, "ImageNet Classification with Deep Convolutional," Advances in Neural Information Processing Systems, pp. 1097-1105, 2012.

[2] C. Szegedy, Wei Liu, Yangqing Jia, P. Sermanet, S. Reed, D. Anguelov, D. Erhan, V. Vanhoucke and A. Rabinovich, "Going deeper with convolutions," Computer Vision and Pattern Recognition (CVPR), pp. 19, 2015.

[3] I. Goodfellow, Y. Bengio and A. Courville, Deep Learning (Adaptive Computation and Machine Learning series), The MIT Press, 2016.

[4] Y. LeCun, L. Bottou, Y. Bengio and P. Haffner, "Gradient-Based Learning Applied to Document Recognition," Proceedings of the IEEE, vol. 86, no. 11, pp. 2278-2324, 1998.

[5] C.-Y. Lee, P. W. Gallagher and Z. Tu, "Generalizing Pooling Functions in CNNs: Mixed, Gated, and Tree," IEEE Transactions on Pattern Analysis and Machine Intelligence, 2017.

[6] F. Agostinelli, M. Hoffman, P. Sadowski and P. Baldi, "Learning Activation Functions To Improve Deep Neural Networks," International Conference on Learning Representations (ICLR), 2015.

[7] N. Srivastava, G. Hinton and A. Krizhevsky, "Dropout: A Simple Way to Prevent Neural Networks from," Journal of Machine Learning Research, no. 15, pp. 1929-1958, 2014. 